\title{
Osteopathische Diagnostik und Therapie bei Leberfunktionsstörungen
}

Die Leber ist als Entgiftungsorgan besonders großen Belastungen ausgesetzt. Vegetativer Stress und metabolische Überbelastung können zu Leberfunktionsstörungen führen. Die Symptome zeigen sich dabei nicht nur in organbezogenen, sondern auch in systemischen Beschwerden. Die erfahrene Osteopathin Magga Corts schildert an einem Beispiel aus ihrer Praxis das Vorgehen bei der osteopathischen Diagnose und Behandlung bei einer viszeralen Dysfunktion der Leber.

Hans B. (49) kommt wegen ständig wiederkehrender Rückenschmerzen in meine Praxis, die Beschwerden traten ca. 6 Monate zuvor erstmals auf. Die Schmerzen finden sich überwiegend im Bereich der Brustwirbelsäule lokalisiert und strahlen in die rechte Thoraxhälfte aus. Sie treten während der Büroarbeit im Sitzen auf, aber auch nachts zwischen 2 und 3 Uhr im Liegen. Die Schmerzen beginnen zunächst sehr milde und steigern sich schleichend. Manchmal werden sie so stark, dass Hans B. nicht mehr sitzen bzw. liegen kann. Wärme lindert, Bewegung hat dagegen wenig Einfluss. Es kommen häufig Kopfschmerzen hinzu.

Hans B. gibt an, dass ihm seine Verdauung zusätzlich Probleme bereitet, er beschreibt sie als „mühsam“. In letzter Zeit fühlt er sich oft sehr müde und energiearm. Zudem erkrankt er bei jeder Erkältungs- und Grippewelle.

\section{Weitere Anamnese}

Hans B. ist $185 \mathrm{~cm}$ groß und wiegt $98 \mathrm{~kg}$. In den vergangenen 2 Jahren hat er insgesamt $10 \mathrm{~kg}$ zugenommen. Die Gewichtszunahme begann, nachdem er das Joggen wegen beruflich bedingtem Zeitmangel komplett eingestellt hatte. Bis dahin ging er $3 \times$ wöchentlich 8-10 km laufen. Hans B.

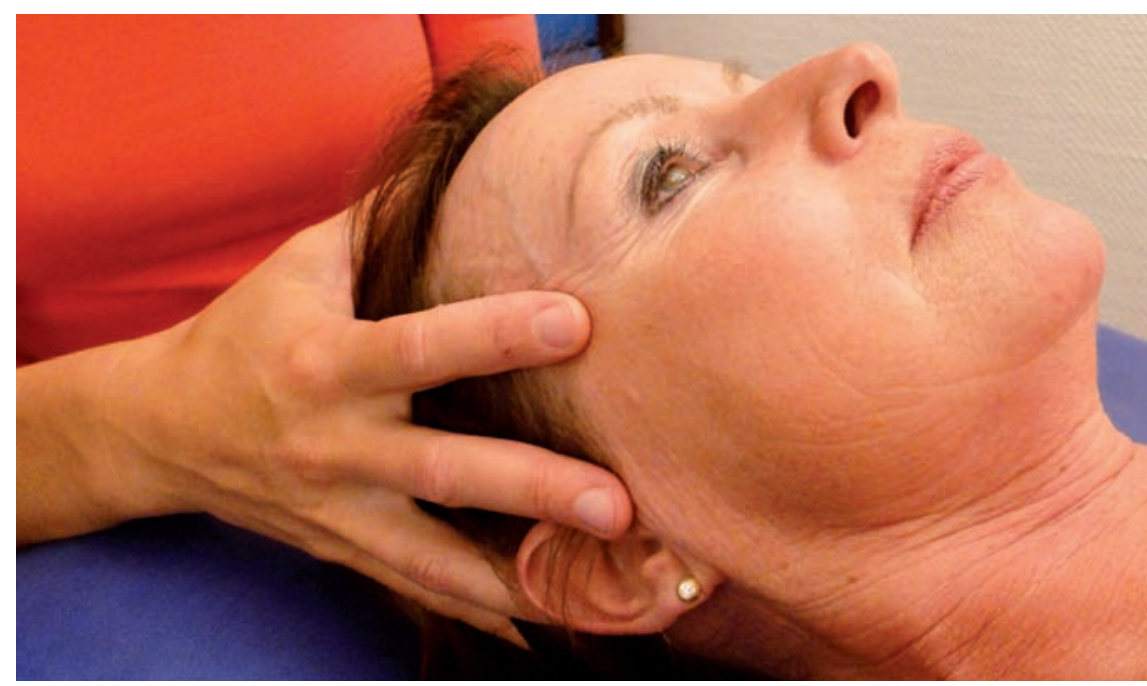

Abb. 1 Beispielhafte Untersuchungssituation: Die Schädeldachhaltung nach Sutherland wird eingenommen, um die Synchondrosis sphenobasilaris (SSB) und dem kraniosakralen Rhythmus zu untersuchen. Foto: (๖) Gert Schäfer (Szene nachgestellt)

arbeitet seit 3 Jahren bei einem Lebensmittelkonzern als Zentraleinkäufer, seine Arbeitszeit beträgt ca. 10 Stunden/Tag.

Anfang des Jahres litt Hans B. unter einem spontan aufgetretenen, sehr schmerzhaften Impingementsyndrom in der rechten Schulter. Kortisonhaltige Injektionen in die Schmerzpunkte im Schulterbereich (Kapselansätze, lange Bizepssehne) linderten die Schmerzen. Die Bewegungseinschränkung blieb jedoch bestehen. Hans B. kann sich erst seit einer F.X. Mayr-Kur wieder uneingeschränkt bewegen. Aktuell liegen außer den Rückenschmerzen keine weiteren körperlichen Einschränkungen bzw. Erkrankungen vor. In der Vorgeschichte finden sich keine Hinweise auf Unfälle oder Operationen.

Eine kürzlich durchgeführte Ultraschalluntersuchung der Gefäße und des Abdomens, die Auskultation der Lunge sowie ein Lungenfunktionstest sind o. B. Hans B. gibt auf Nachfrage an, dass ihm forciertes tiefes Durchatmen in beruflichen Stresssituationen schwerfällt.
Ein ebenfalls kürzlich bei Hans B. durchgeführtes Belastungs-EKG zeigt eine verminderte Ausdauerleistungsfähigkeit ohne Herzrhythmusstörungen. Der Blutdruck beträgt 140/90 mm Hg. Etwa 6 Wochen alte Laborbefunde zeigen erhöhte Triglyzeride, ein Gesamt-Cholesterin von $240 \mathrm{mg} / \mathrm{dl}$ sowie einen grenzwertig erhöhten GGT-Wert. Die Blutuntersuchung bestätigt einen ausreichenden Impfschutz gegen Hepatitis B, eine Hepatitis-C-Infektion ist nicht nachweisbar. Urin- und Stuhluntersuchung sind ebenfalls $\mathrm{o}$. B.

Hans B. nimmt keine Medikamente ein, außer gelegentlich ASS gegen die Kopfschmerzen. Er raucht nicht, trinkt jedoch viel Kaffee und mäßig viel Alkohol. Wasser, Saft etc. trinkt er insgesamt den Tag über zu wenig. Hans B. isst Mischkost, meist unregelmäßig und in Hektik. Deftiges Essen, das er häufig spät abends zu sich nimmt, und Alkohol bereiten ihm ein unangenehmes Völlegefühl im oberen Abdomen. Familiär besteht Neigung zur Adipositas. 


\section{Diagnostisches Vorgehen}

Aus osteopathischer Sicht gilt in der Diagnostik unter Berücksichtigung der Anamnese folgender Leitgedanke: Die Untersuchung wird erst global durchgeführt, um dann zur lokalen spezifischen Untersuchung des maßgeblich in Dysfunktion befindlichen Gewebes zu kommen. Ziel ist es, den Bereich der größten Spannung festzustellen, weil diese Spannungszone den Körper zwingt, sich neu zu organisieren.

\section{Untersuchung im Stehen}

Ich beginne mit einer globalen osteopathischen Untersuchung in mehreren Positionen, um das Muster der Dysfunktion (Ursache und systemische Wirkung) insgesamt erfassen zu können. Zunächst erfolgt dazu eine Inspektion im Stand.

Hans B. zeigt eine in der BWS gebeugte Haltung, das Haltungsmuster entspricht dem anterioren Typus. Mir fällt die Leberzone im Bereich des Dermatoms Th 6-10 rechts posterior auf. Sie ist etwas verquollen. Der Kopf von Hans B. ist in leichter Extension nach links rotiert und es besteht eine Seitneigung nach rechts. An seiner rechten Schulter fällt mir eine Rotation nach innen auf. Bei genauerem Betrachten sehe ich, dass Hans B.s Becken gekippt ist, das rechte Bein steht in Außenrotation und die Fußlängsgewölbe neigen zum KnickSenkfuß.

Nach der Inspektion erfolgen jetzt Funktionstests im Stand. Ich beginne mit dem Ecoute-Test. Hierbei achte ich auf die Eigenbewegung des Körpers, die Faszienspannung zieht den Körper in Richtung des Bereichs, in dem die Spannung am größten ist. Bei Hans B. ist es eine Eigenbewegung nach anterior, Zeichen für eine viszerale Dysfunktion in Thorax, Abdomen oder kleinem Becken. Gerade beim anterioren Haltungstypus ist eine viszero-somatische Verkettungsreaktion zu beachten: Eine Organdysfunktion kann über neurophysiologische Verbindungen zu Beschwerden im parietalen System führen. Die Leber wird sympathisch aus den thorakalen Wirbelsäulensegmenten Th 5-9 (6-10) versorgt, eine Reizung des Organs führt daher zur Fazilitation des Segments. Das kann Blockaden auslösen.

\section{Weitere Bewegungstests}

An die Funktionstests im Stand schließen sich Tests zur Beurteilung der aktiven und

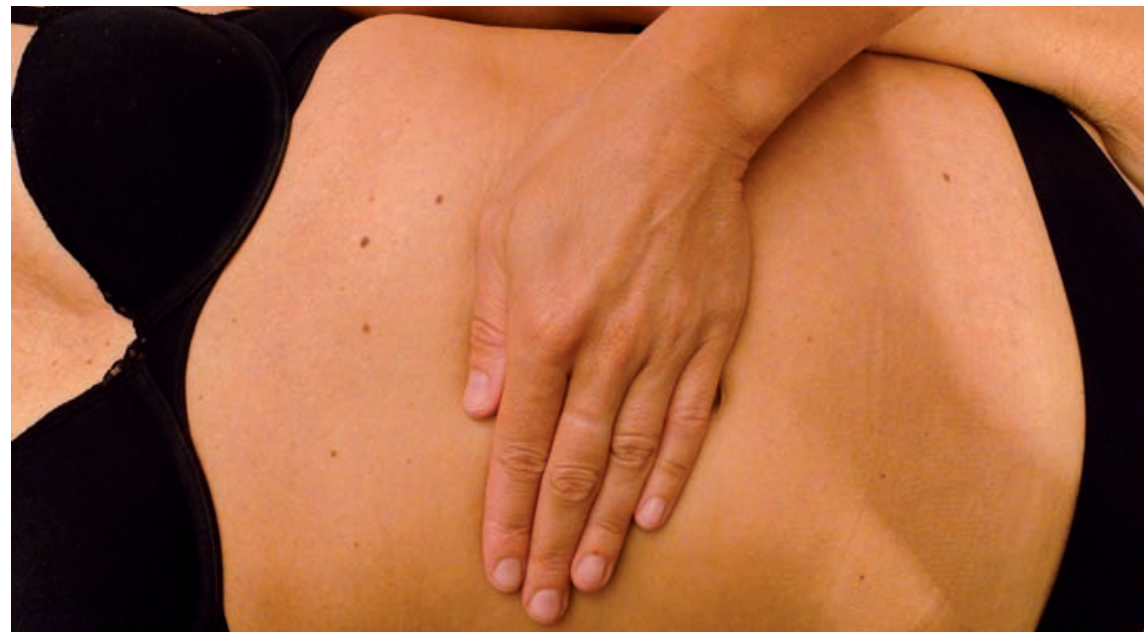

Abb. 2 Beispielhafte Untersuchungssituation: Ist das abdominale Diaphragma unter Spannung? Dieser fasziale Test gibt weitere Informationen. Foto: (c) Gert Schäfer (Szene nachgestellt)

passiven Wirbelsäulenbewegung in Flexion, Extension, Seitneigung und Rotation an. Der Hip-Drop-Test erlaubt es mir, die Seitneigung der BWS, LWS und im lumbosakralen Übergang zu beurteilen. Der Vorlauftest ermöglicht es, eine Asymmetrie oder einschränkende mechanische Funktion im Beckenring zu erkennen (Verhältnis Ileumbewegung zur Sakrumbewegung). Bei Hans B. deuten diese Tests auf eine eingeschränkte Extensionsfähigkeit in der BWS und eine verminderte Rotationsfähigkeit der HWS nach rechts.

\section{Untersuchung im Sitzen}

Um das Ergebnis des Ecoute-Tests im Stand zu bestätigen, erfolgt der Test jetzt noch einmal im Sitzen. Hierbei fällt eine Rotoskoliose auf (Wirbelsäulenverkrümmung in der Frontalebene). Auch den Vorlauftest führe ich bei Hans B. noch einmal im Sitzen durch und vergleiche ihn mit dem Testergebnis im Stand. Er ist positiv, die Sakrumbewegung ist im Verhältnis zur Ileumbewegung eingeschränkt. Ursache dafür ist häufig eine absteigende Kraftkette, die die Mechanik des Sakrums stört. Da der Test bei Hans B. nur im Sitzen positiv ausfällt, haben die unteren Extremitäten keinen Einfluss auf das Dysfunktionsbild.

Um die segmentale Störung in der Wirbelsäule näher zu untersuchen, lasse ich Hans B. den Vorlauftest nur in Flexion und ohne Mitbewegung in der HWS durchführen. Der Test ist positiv. Wäre er negativ, bestünde ein Problem im HWS-Bereich. Anschließend bitte ich Hans B., den Test noch einmal mit retraktierten Schulter- blättern zu wiederholen. So kann ich differenzieren, ob die Ursache im lumbalen oder thorakalen Bereich zu suchen ist. Der Test fällt negativ aus, was auf eine Ursache im thorakalen Bereich hinweist.

\section{Prüfung der Respiration}

Als Nächstes überprüfe ich die Atembewegungen. Hierzu lege ich meine Hände Hans B. zunächst auf die Rippen, dann auf das Sternum und schließlich auf die oberen Rippen/den oberen Schultergürtel auf. Ich achte sowohl auf die Beweglichkeit von Zwerchfell, endothorakalen Faszien und Sternum bei der Ein- und Ausatmung als auch auf die Beweglichkeit der oberen Rippen und zervikopleuralen Aufhängestrukturen. Zwerchfell und endothorakale Faszien zeigen sich nur gering beweglich.

\section{Untersuchung in Rückenlage}

Jetzt erfolgen Untersuchungen in der Rückenlage. Ich beginne mit der Testung der Synchondrosis sphenobasilaris (SSB) und des kraniosakralen Rhythmus, indem ich mit den Händen die Schädeldachhaltung nach Sutherland einnehme( Abb. 1, S. 26). Hierbei kann ich eine eingeschränkte Beweglichkeit der SSB und eine fasziale Hypertension der Zentralsehne feststellen.

Als Nächstes erfolgt eine Testung des kraniozervikalen Diaphragmas. Dabei untersuche ich ossäre, muskuläre, ligamentäre und fasziale Strukturen am Übergang von Okziput und Atlas. Bei Hans B. lässt sich eine rechtsseitige Verspannung des Diaphragmas feststellen, die den N. vagus negativ beeinflussen kann. 


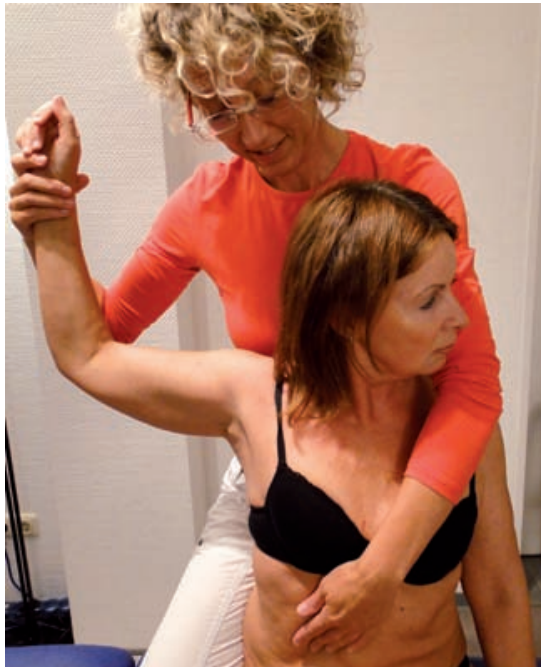

Abb. 3 Beispielhafte Untersuchungssituation: Sotto-Hall-Test: Ist die Leber die primäre Dysfunktion? Dieser Test gibt Aufschluss.

Foto: @ Gert Schäfer (Szene nachgestellt)

Der passive Bewegungstest der HWS ergibt eine Dysfunktion im Segment C 3/4 (Extension/Rotation links, Seitneige rechts).

Die differenzierte myofasziale Testung von Thorax, Abdomen und kleinem Becken zeigt im rechten oberen Quadranten des Abdomens eine erhöhte Spannung (Leber, Gallenblase). Das abdominale Diaphragma (- Abb. 2, S. 27) weist eine rechtsseitige Festigkeit auf, der rechte Thorax ist unelastisch. Es besteht zudem eine Spannung im M. psoas major rechts, was eine Außenrotation des Beines zur Folge hat. Der fasziale Tonus des Diaphragma pelvis ist erhöht.
Safety-Test (Leberrandpalpation) und Murphy-Test sind unauffällig, der Mobilitätsund Motilitätstest der Leber nach Barral zeigt dagegen eine eingeschränkte Organbewegung in allen Ebenen sowie eine Fixation des Organs.

\section{Untersuchung in Bauchlage}

Der passive Test der thorakal-lumbalen Wirbelsäule mit der Maidstone oscillatory technique (rhythmische Schaukelbewegung des Sakrums mit gleichzeitiger Segmenttestung der Wirbelsäule) ergibt eine Flexionsdysfunktion rechts in Th $7 / 8$.

\section{Sotto-Hall-Kontrolltest für die Leber}

Um meinen Befund (Leber primär in Dysfunktion, BWS-Segmentblockade ist nur sekundär) abzusichern, führe ich den Sotto-Hall-Test (nach Barral) durch ( Abb. 3). Mit diesem Test prüfe ich, ob die Leber die primäre Dysfunktion ist.

Die hohe fasziale Spannung, die von einem Organ in Dysfunktion ausgehen kann, wird hierbei in Bezug auf den Radialis-Puls getestet. Man bringt dazu das thorakale fasziale System unter Spannung, indem man den gebeugten Arm des Patienten in Abduktion und Außenrotation bringt. Der Kopf ist dabei zur Gegenseite (links) rotiert. Verschwindet der Puls in dieser Position, ist der Test positiv. Dass der Puls nicht mehr tastbar ist, liegt an der erhöhten Spannung der Faszien in der Fossa supraclavicularis major, die die A. subclavia abdrückt und dadurch den Puls beeinträchtigt. Die osteopathische Dysfunktion der Leber (fixiertes Organ) bringt über die fasziale Kette Spannung bis in die obere Thoraxapertur. Man ergänzt den Test, indem man das Organ anhebt und die fasziale Kette dadurch entspannt. Kehrt der Puls zurück, ist das Organ ursächlich für die Dysfunktion. Das trifft bei Hans B. zu.

\section{Diagnose}

Ich diagnostiziere bei Hans B. eine primäre viszerale Dysfunktion der Leber in der Motilität und Mobilität in allen 3 Ebenen (Adhäsion). Die BWS-Schmerzen resultieren aus einem fazilitierten Segment (Flexionsdysfunktion rechts in Th 7/8). Mögliche Auslöser sind eine metabolische Überreizung und vegetativer Stress.

\section{Behandlung}

Die Leber-Adhäsion hat eine komplexe Wirkung auf das Körpersystem. Daher ist folgende Reihenfolge in der Behandlung angezeigt. Die Behandlung wird in wöchentlichen Abständen wiederholt und erfolgt ca. 8-mal. Eine Testung des Organsystems in den einzelnen Behandlungen ist immer wieder nötig, um den Status quo zu erfassen und ggf. die Behandlungsschwerpunkte zu verändern.

\section{Systemische Behandlung}

Zunächst entspanne ich das kraniozervikale Diaphragma. Durch die Entspannung normalisieren sich die Funktionen des Nervensystem, insbesondere des N. vagus (parasympathische Regulation der Leber), und die sensible Versorgung der Dura mater

Weitere Indikationen für eine osteopathische Behandlung der Leber

- Adhäsion der Leber (Befreiung der Gleitschichten)

- Abwehrschwäche des Immunsystems, eingeschränkte Stoffwechselaktivität, mangelnde Vitalität (Verbesserung der Trophik, Normalisierung des Stoffwechsels)

- unklare Schultergelenkbeschwerden (vorwiegend rechts)

- Verminderung von rezidivierenden Wirbelblockaden

n positiver Einfluss auf den mentalen/ psychischen Zustand
Kontraindikationen für eine osteo-

pathische Behandlung der Leber

- akute Entzündung

- Ikterus

- Gallensteine

- Leberzirrhose

- Hepatomegalie

- allgemeine Herzinsuffizienz

- Tumoren

- Splenomegalie

Differenzialdiagnose (DD):

- akute, chronische Hepatitis

- Fettleber

- Leberzirrhose

- Leberabszess
- Lebermetastasen

- hepatorenales Syndrom

- Leberschäden bei Stoffwechselerkrankungen

DD systemisch:

- Rechtsherzinsuffizienz > Stauungsleber

v Portale Hypertension (Pfortaderhochdruck)

- Hypo-, Hyperthyreose

- Diabetes mellitus

- Morbus Cushing (Funktionsstörung der Nebennieren)

- Anorexia nervosa, Bulimia nervosa

- Alkohol, Medikamente (Toxine) 
(sie wird innnerviert aus den Segmenten C 1-3). Das reduziert den Kopfschmerz.

Danach erfolgt eine Mobilisation der Segmente C 3/4 mittels Muskelenergietechniken. Durch die Zwerchfellspannung und die Kapselreizung der Leber kommt es über den versorgenden N. phrenicus zu einer Fazilitation der Segmente C 3-5. Bei Extrembelastung kann das Schmerzsyndrome in der rechten Schulter auslösen, da die nervale Versorgung der Schulter aus den gleichen Segmenten entspringt (Neigung zum Impingementsyndrom).

Anschließend weite ich die obere Thoraxapertur mit Muskelenergietechniken für die Mm. scalenii, den M. trapezius und M. sternocleidomastoideus sowie den M. subclavius. Zusätzlich erfolgt eine manuelle Mobilisation der 1. Rippe sowie der Clavicula. Das führt zu einer verbesserten hämatogenen Zirkulation (Hans B. neigt zu milder Hypertonie und Kopfschmerzen).

Zum Schluss entspanne ich die endothorakale Faszie, mobilisiere das Mediastinum und die Interkostalräume, um dadurch eine fasziale Entspannung des Thorax zu bewirken, und normalisiere die abdominale Zwerchfellspannung mittels Dehntechniken bzw. Rebound-Technik. Alles zusammen verbessert die hämodynamische Regulation der Gewebe, die Druckverhältnisse in Thorax und Abdomen harmonisieren sich (Hans B. hat unter Stress Mühe, tief in das Zwerchfell einzuatmen).

\section{Behandlung der Organaufhängung}

Es erfolgt eine indirekte Mobilisation der Leber: in der Frontalebene über die Rippen zur Spannungsminderung im Lig. triangulare dextrum, in der Sagittalebene über die indirekte Rippentechnik zur Mobilisation des Lig. coronarium und in der Transversalebene um die Bewegungsachse (V. cava). Auch die indirekte Technik für die Motilität der Leber nach Barral wende ich an.

Das Lig. hepatoduodenale wird für eine verbesserte Hämodynamik der Leber gedehnt, im Ligament befinden sich die 3 wichtigen zirkulatorischen Strukturen: V. portae hepatis, A. hepatica propria und der Ductus choledochus. Anschließend mobilisiere ich die Gleitfläche zum Colon transversum zur Besserung der Obstipation.

\section{Behandlung weiterer Strukturen} Eine Entlastung des fazilitierten Segments Th $7 / 8$ durch Gelenkmanipulation sowie ein Ausgleich des Vegetativums über die Befreiung des Foramen jugulare (Austritt N. vagus) mit abschließender Harmonisierung des kraniosakralen Systems durch Duraschaukel schließen die Behandlung ab.

\section{Wie geht es Hans B. heute?}

Hans B. ist heute schmerzfrei. Die osteopathische Behandlung hat die Mobilität und die Motilität seiner Leber wiederhergestellt und das Körpersystem harmonisiert. Hans B. weiß aber durch die therapeutische Beratung auch, dass dieser Zustand nur erhalten bleibt, wenn er sein Ernährungsverhalten ändert und Stress abbaut. Ich habe Hans B. ergänzend zur osteopathischen Behandlung einen Ernährungsplan sowie Tipps zur regelmäßigen Bewegung und zum Stressmanagement mit nach Hause gegeben.

Dieser Artikel ist online zu finden unter: http://dx.doi.org//10.1055/s-0030-1268046

\section{(1) Weiterführende Literatur}

[1] de Coster M, Pollaris, A. Viszerale Osteopathie. Stuttgart: Hippokrates; 2007

[2] Hebgen E. Viszeralosteopathie - Grundlagen und Techniken. Stuttgart: Hippokrates; 2004

[3] Hebgen E. Checkliste Viszerale Osteopathie Stuttgart: Hippokrates; 2009

[4] Liem T, Dobler, TK. Leitfaden Osteopathie. München: Elsevier; 2000

[5] Liem T, Dobler TK, Puylaert M (Hrsg.). Leitfaden Viszerale Osteopathie. München: Elsevier; 2005

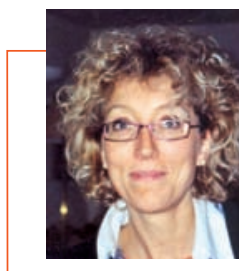

HP Magga Corts Elisenstr. 1 50667 Köln

Magga Corts ist Heilpraktikerin, Osteopathin und diplomierte Sportwissenschaftlerin. Nach langjähriger Tätigkeit in der Arbeits- und Sportmedizin betreibt sie heute eine Praxis für Osteopathie und Naturheilkunde in Köln. Sie ist Dozentin für ausgewählte Themen in der Osteopathie, Chiropraktik, Neuraltherapie und leitet zusammen mit Ina ter Harmsel das ACON-COLLEG.

E-Mail: magga.corts@praxis-corts.de 